\title{
Ride Sharing \& Data Privacy: How Data Handling Affects the Willingness to Disclose Personal Information
}

\author{
Carsten Hesselmann ${ }^{1}$, , Jan Gertheiss ${ }^{2}$ (D) b , Jörg P. Müller ${ }^{1 ~ c}$ \\ ${ }^{1}$ Institute of Informatics, Clausthal University of Technology, ${ }^{2}$ School of Economics and Social Sciences, Helmut Schmidt University \\ Keywords: privacy, ride sharing, data disclosure, sharing economy \\ https://doi.org/10.32866/001c.29863
}

Digital services like ride sharing rely heavily on personal data, e.g. name or age, as individuals have to disclose personal information in order to gain access to the market. This information is exchanged with other participants; yet, the service provider usually gives little to no information regarding the privacy status of the disclosed data. To further investigate the implications of this, we conducted an online survey asking participants about their willingness to disclose personal information under varying privacy status. The results of 127 complete responses show that differences in information privacy settings influence the participants' willingness in a significant way.

\section{Questions}

Privacy is important when an individual interacts with others as their social circles and boundaries affect the perceived privacy (Page, Kobsa, and Knijnenburg 2012; Squicciarini et al. 2014; Jagadish 2020). In the sharing economy - a term for (digital) peer-to-peer marketplaces where individuals share their private property with others - communicating and meeting with unknown persons is a common interaction. Individuals have varying motivations for sharing (Schor et al. 2016; Milanova and Maas 2017) and there are numerous marketplaces which offer services for sharing, for example, apartment rentals and shared rides. Privacy is a significant factor when individuals decide about participating in such a shared marketplace (Ranzini et al. 2017). Despite the importance of information privacy and its regulation, e.g. (General Data Protection Regulation 2016), providers of such marketplaces rarely provide information to the participants regarding the privacy of their personal data, which is a central issue for any digital service. We investigate how this lack of transparency could affect user's participation.

Therefore, we previously analyzed twelve ride sharing services offered in Germany, Austria, and Switzerland to identify their data practices in regards to data privacy and privacy-related features (Hesselmann, Gertheiss, and Müller 2021). Table 1 shows the collected and exposed (towards other users) data attributes, e.g. name or address, across the analyzed ride sharing services, illustrating the diversity of the data practices and lack of standardization

\footnotetext{
a 38678 Clausthal-Zellerfeld, Germany

b 22043 Hamburg, Germany 
Table 1.

\begin{tabular}{|c|c|c|c|c|c|c|c|c|c|c|c|c|}
\hline & (1) & (2) & (3) & (4) & (5) & (6) & (7) & (8) & (9) & (10) & (11) & (12) \\
\hline collected data & 9 & 23 & 13 & 16 & 14 & 5 & 6 & 16 & 11 & 30 & 15 & 13 \\
\hline - mandatory & 3 & 14 & 3 & 9 & 4 & 4 & 3 & 2 & 8 & 4 & 8 & 9 \\
\hline - optional & 6 & 9 & 10 & 7 & 10 & 1 & 3 & 14 & 3 & 26 & 7 & 4 \\
\hline exposed data & 9 & 11 & 11 & 11 & 11 & 5 & 4 & 15 & 5 & 18 & 8 & 10 \\
\hline - profile data & 0 & 2 & 0 & 1 & 3 & 0 & 0 & 0 & 0 & 5 & 0 & 0 \\
\hline - ride offer & 9 & 4 & 6 & 5 & 3 & 5 & 4 & 7 & 0 & 11 & 8 & 10 \\
\hline - both pages & 0 & 5 & 5 & 5 & 5 & 0 & 0 & 8 & 5 & 2 & 0 & 0 \\
\hline
\end{tabular}

among services. The results of the analysis served as basis for the online survey in which we asked participants to state their willingness to disclose personal information under varying privacy conditions. We defined the following research questions for our survey:

- To what degree does the exposure of personal information towards other users influence an individual's willingness to disclose it?

- How strongly is the willingness to disclose personal information influenced by the availability of privacy settings (e.g. change exposure of certain information to other users)?

- To what extent does the validation of the authenticity of personal information influence an individual's willingness to disclose personal information?

\section{Methods}

The survey was advertised within the Clausthal University of Technology as well as topic- and field-related mail channels in Germany and includes 127 complete responses. Due to the chosen recruitment methods for our study, participants are predominantly young adults which also form the most common age group to participate in shared rides (Schoesslers 2016; European Federation for Transport and Environment 2020). Most participants live in Germany (89\%). $61 \%$ of participants are male and $72 \%$ report prior experience with ride sharing.

We included a set of 15 data attributes, mixed with common and rare data from our prior analysis of ride sharing services, and combined this with four conditions:

1. The personal information is visible for other users

2. The personal information is not visible for other users 
How fair do you consider it for the operator of a ride sharing service to ask you for the following information, when your information is not visible to other users?

very unfair unfair

date of birth

profile picture somewhat somewhat unfair fair

fair very fair

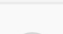

Figure 1. Example of a survey question with six-point Likert scale

3. Profile settings are available to manage the visibility

4. The authenticity of personal information is validated by the service provider but the information is not disclosed to other users

Instead of focusing on privacy concerns (which are often reported to be high but rarely influence the actual disclosure behavior of an individual significantly (Lutz et al. 2018)), we asked participants to rate fairness and sensitivity of data disclosure in the context of ride sharing, as these factors are good predictors of actual disclosure behavior (Malheiros, Preibusch, and Sasse 2013). We conducted this as a within-subject study, i.e. all participants rated every condition. Based on Malheiros, Preibusch, and Sasse (2013), fairness was measured with ratings ranging from very unfair $(0)$ to very fair $(+5)$ and sensitivity was measured as the tendency of data disclosure from very unlikely (0) to very likely $(+5)$. To account for ordering effects, the sequence in which the questions were presented to the participant was randomized. An example of a survey question is displayed in Figure 1 .

\section{Findings}

We used the condition (i.) personal information is visible for other users as a baseline to compare with the other conditions as it was the most common in our analysis of ride sharing services (Hesselmann, Gertheiss, and Müller 2021). Thus, we conducted three comparisons A, B, and C:

1. (i.) Information visible compared with (ii.) information not visible

2. (i.) Information visible compared with (iii.) profile setting available

3. (i.) Information visible compared with (iv.) validation available

The p-values are obtained from paired (two-sided) t-tests. To account for multiple testing, we used the Bonferroni-Holm-Method (Holm 1979) to address the Family Wise Error Rate. The differences in mean ratings are given 
Baseline for comparison: mean ratings of the condition (i) information visible

\begin{tabular}{|c|c|c|c|c|c|c|c|c|c|c|c|c|c|c|c|}
\hline & 3,91 & 2,79 & 3,11 & 0,98 & 2,31 & 1,24 & 2,70 & 2,87 & 2,46 & 0,81 & 1,04 & 1,23 & 3,93 & 1,99 & 3,50 \\
\hline & 3,83 & 2,63 & 3,11 & 0,94 & 2,05 & 1,07 & 2,47 & 2,67 & 2,36 & 0,72 & 0,94 & 1,12 & 3,69 & 1,77 & 3,46 \\
\hline \multirow{4}{*}{ A } & $-0,02$ & 0,70 & $-0,05$ & $-0,02$ & 0,63 & 1,20 & 0,79 & $-0,54$ & 0,72 & 0,13 & 1,44 & 0,04 & $-0,35$ & 0,75 & $-0,42$ \\
\hline & $(0,7974)$ & $(0,0000)$ & $(0,6990)$ & $(0,8882)$ & $(0,0000)$ & $(0,0000)$ & $(0,0000)$ & $(0,0001)$ & $(0,0000)$ & $(0,1614)$ & $(0,0000)$ & $(0,6934)$ & $(0,0027)$ & $(0,0000)$ & $(0,0020)$ \\
\hline & $-0,25$ & 0,43 & $-0,13$ & 0,17 & 0,61 & 1,07 & 0,36 & $-0,50$ & 0,54 & 0,16 & 1,24 & $-0,01$ & $-0,29$ & 0,59 & $-0,37$ \\
\hline & $(0,0085)$ & $(0,0036)$ & $(0,3211)$ & $(0,1231)$ & $(0,0000)$ & $(0,0000)$ & $(0,0222)$ & $(0,0000)$ & $(0,0000)$ & $(0,0305)$ & $(0,0000)$ & $(0,9303)$ & $(0,0025)$ & $(0,0000)$ & $(0,00 C$ \\
\hline \multirow{4}{*}{ B } & 0,16 & 0,57 & 0,36 & 0,39 & 0,67 & 0,72 & 0,85 & 0,52 & 0,35 & 0,40 & 1,06 & 0,57 & 0,00 & 0,77 & 0,33 \\
\hline & $(0,0840)$ & $(0,0000)$ & $(0,0018)$ & $(0,0056)$ & $(0,0000)$ & $(0,0000)$ & $(0,0000)$ & $(0,0000)$ & $(0,0020)$ & $(0,0010)$ & $(0,0000)$ & $(0,0001)$ & $(1,0000)$ & $(0,0000)$ & $(0,002$ \\
\hline & 0,26 & 0,63 & 0,44 & 0,34 & 0,90 & 0,90 & 0,72 & 0,56 & 0,35 & 0,17 & 0,96 & 0,35 & 0,18 & 0,86 & 0,27 \\
\hline & $(0,0013)$ & $(0,0000)$ & $(0,0001)$ & $(0,0024)$ & $(0,0000)$ & $(0,0000)$ & $(0,0000)$ & $(0,0000)$ & $(0,0009)$ & $(0,0552)$ & $(0,0000)$ & $(0,0011)$ & $(0,0101)$ & $(0,0000)$ & $(0,000$ \\
\hline \multirow{4}{*}{ C } & $-0,92$ & 0,20 & $-0,12$ & 2,01 & 0,69 & 1,75 & 0,88 & $-0,03$ & 0,90 & 0,11 & 1,66 & 2,35 & $-1,28$ & 0,65 & $-0,66$ \\
\hline & $(0,0000)$ & $(0,2309)$ & $(0,4980)$ & $(0,0000)$ & $(0,0001)$ & $(0,0000)$ & $(0,0000)$ & $(0,8224)$ & $(0,0000)$ & $(0,2875)$ & $(0,0000)$ & $(0,0000)$ & $(0,0000)$ & $(0,0002)$ & $(0,000$ \\
\hline & $-1,31$ & $-0,10$ & $-0,58$ & 1,59 & 0,48 & 1,46 & 0,70 & $-0,02$ & 0,50 & 0,19 & 1,39 & 2,06 & $-1,31$ & 0,60 & $-0,81$ \\
\hline & $(0,0000)$ & $0,5007)$ & $(0,0025)$ & $(0,0000)$ & $(0,0085)$ & $(0,0000)$ & $(0,0000)$ & $(0,9120)$ & $(0,0020)$ & $(0,0591)$ & $(0,0000)$ & $(0,0000$ & 0,0000 & 0,0002 & $(0,000$ \\
\hline & 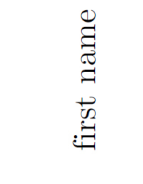 & 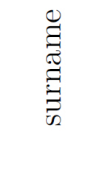 & $\frac{\dot{\theta}}{\vec{D}}$ & 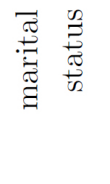 & 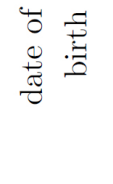 & $\frac{D_{0}}{0}$ & 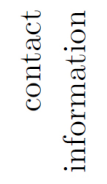 & 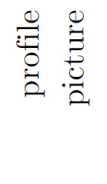 & 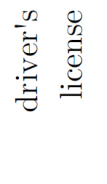 & $\begin{array}{l}\overrightarrow{0} \\
\stackrel{0}{0} \\
\frac{0}{2} \\
\overrightarrow{0}\end{array}$ & $\begin{array}{l}\frac{\pi}{\pi} \\
\frac{\pi}{0} \\
\frac{0}{0} \\
\frac{x}{\pi} \\
\tilde{\pi}\end{array}$ & 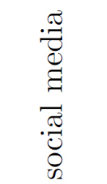 & 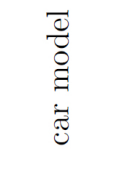 & 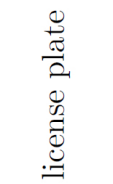 & 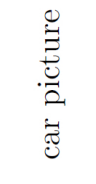 \\
\hline
\end{tabular}

Table 2. Differences in mean ratings for Fairness $(\mathrm{F})$ and Sensitivity $(\mathrm{S})$ and $\mathrm{p}$-values in parentheses, with bold p-values indicating significance $($ alpha $=0.05)$ after Bonferroni-Holm adjustment

in Table 2, with positive sign indicating a favorable rating for the latter condition of the respective comparison. The corresponding $\mathrm{p}$-value is given in parentheses below the difference in mean rating.

The differences in comparison $\mathrm{A}$ indicate that users prefer their information to be made accessible to other users, e.g. for the data attributes profile picture, car type/model, and car picture, and are more inclined to disclose the information. However, for the remaining data attributes users are equally or less inclined to disclose their information as mean ratings are equal or in favor of information being not visible. In contrast to this, the results of comparison $B$ reveal a onesided preference in favor of profile settings. A look at the mean ratings shows that profile settings increase perceived fairness and/or the likelihood of data disclosure for all 15 data attributes. Comparison $\mathrm{C}$ displays that information validation has varying effects on the user. It affects the perceived fairness and likelihood in a positive way for bank details and in a negative way for first name.

When looking at the data attributes across the conducted comparisons, gender and employer show only minimal differences in mean ratings. The attribute social media is an interesting result as participants strongly prefer to validate their profile but are reluctant to share it with other users. The opposite effect is seen for first name; the mean values are almost identical across comparison $\mathrm{A}$ and $\mathrm{B}$ but change strongly for $\mathrm{C}$, which shows that participants have little concern to disclose their name but are reluctant to validate it via a copy of their ID. A look at the p-values shows that 54 hypotheses were rejected, indicating significant differences in $60 \%$ of the conducted comparisons.

Overall the results of this preliminary study are promising for the direction in which our work is heading and we will continue our efforts and improve upon the small data set with additional experiments ideally seconding our approach. 
Submitted: October 19, 2021 AEDT, Accepted: November 09, 2021 AEDT

This is an open-access article distributed under the terms of the Creative Commons Attribution 4.0 International License (CCBY-SA-4.0). View this license's legal deed at https://creativecommons.org/ licenses/by-sa/4.0 and legal code at https://creativecommons.org/licenses/by-sa/4.0/legalcode for more information. 


\section{REFERENCES}

European Federation for Transport and Environment. 2020. "Future of Uber in Europe: Electric and Shared? Consumer Attitudes towards Uber and Taxi Services.”

General Data Protection Regulation. 2016. Regulation (EU) 2016/679 of the European Parliament and of the Council of 27 April 2016 on the Protection of Natural Persons with Regard to the Processing of Personal Data and on the Free Movement of Such Data, and Repealing Directive 95/46/EC. http://data.europa.eu/eli/reg/2016/679/2016-05-04.

Hesselmann, C, J Gertheiss, and J.P Müller. 2021. "Ride Sharing \& Data Privacy: An Analysis of the State of Practice." https://arxiv.org/abs/2110.09188.

Holm, S. 1979. "A Simple Sequentially Rejective Multiple Test Procedure.” Scandinavian Journal of Statistics 6 (2): 65-70. https://www.jstor.org/stable/4615733.

Jagadish, H. V. 2020. “Circles of Privacy.” Journal of Consumer Psychology 30 (4): 774-79. https://doi.org/10.1002/jcpy.1188.

Lutz, Christoph, Christian Pieter Hoffmann, Eliane Bucher, and Christian Fieseler. 2018. "The Role of Privacy Concerns in the Sharing Economy." Information, Communication E'Society 21 (10): 1472-92. https://doi.org/10.1080/1369118x.2017.1339726.

Malheiros, M., S. Preibusch, and M.A. Sasse. 2013. "Fairly Truthful": The Impact of Perceived Effort, Fairness, Relevance, and Sensitivity on Personal Data Disclosure.” In Trust and Trustworthy Computing, edited by D. Hutchison, 7904:250-66. Of Lecture Notes in Computer Science. Berlin, Heidelberg: Springer Berlin Heidelberg. https://doi.org/10.1007/978-3-642-38908-519.

Milanova, V., and P. Maas. 2017. "Sharing Intangibles: Uncovering Individual Motives for Engagement in a Sharing Service Setting.” Journal of Business Research 75: 159-71. https://doi.org/ 10.1016/j.jbusres.2017.02.0025.

Page, X., A. Kobsa, and B. Knijnenburg. 2012. "Don't Disturb My Circles! Boundary Preservation Is at the Center of Location-Sharing Concerns." Proceedings of the International AAAI Conference on Web and Social Media 6 (1): 266-73. https://ojs.aaai.org/index.php/ICWSM/article/view/14277.

Ranzini, Giulia, Michael Etter, Christoph Lutz, and Ivar E. Vermeulen. 2017. "Privacy in the Sharing Economy.” SSRN Electronic Journal. https://doi.org/10.2139/ssrn.2960942.

Schoesslers. 2016. "Carjump Veröffentlicht Forsa-Umfrage Zu Carsharing in Großstädten.” 2016. https://schoesslers.com/carjump-veroeffentlicht-forsa-umfrage-zu-carsharing-in-grossstaedten/.

Schor, J. et al. 2016. "Debating the Sharing Economy." Journal of Self-Governance and Management Economics 4 (3): 7-22.

Squicciarini, Anna, Sushama Karumanchi, Dan Lin, and Nicole DeSisto. 2014. "Identifying Hidden Social Circles for Advanced Privacy Configuration.” Computers E'Security 41 (March): 40-51. https://doi.org/10.1016/j.cose.2013.07.007. 